\title{
Chronic kidney disease and subclinical lacunar infarction are independently associated with frontal lobe dysfunction in community-dwelling elderly subjects: the Sefuri brain MRI study
}

\author{
Hiroshi Yao ${ }^{1}$, Yoshikazu Miwa ${ }^{2}$, Yuki Takashima ${ }^{1}$, Koji Yahara ${ }^{3,4,6}$, Manabu Hashimoto $^{1}$, Akira Uchino ${ }^{5}$, \\ Takefumi Yuzuriha $^{1}$ and Toshiyuki Sasaguri ${ }^{2}$
}

\begin{abstract}
Although recent studies have found that chronic kidney disease (CKD) is an independent risk factor for cognitive impairment in population-based cohorts, the mechanisms of cognitive impairment in subjects with CKD are unclear. We examined 503 elderly subjects (mean age: 72.4 years), who were living independently at home without apparent dementia, using MRI. The subject was judged as having frontal lobe dysfunction if the scores on the modified Stroop test were higher than the fifth quintile for each given decade. Serum creatinine values, measured by the enzymatic method, were used for the Japanese equation of estimated glomerular filtration rate (eGFR). Subjects in the frontal lobe dysfunction group tended to have higher blood pressure, lower eGFR and more lacunar infarcts, and were less educated. When possible confounders were entered into the multivariate logistic regression model, the independent predictors of frontal lobe dysfunction were eGFR (odds ratio $0.854 ; 95 \%$ confidence interval (Cl) $0.743-0.983$ per $10 \mathrm{ml} \mathrm{min}^{-1}$ per $1.73 \mathrm{~m}^{2}$ ) and the number of lacunar infarction (odds ratio $1.460 ; 95 \% \mathrm{CI}$ 1.127-1.892). The mean of the logarithmically transformed Stroop test scores in the eGFR $<60 \mathrm{ml} \mathrm{min}-1$ per $1.73 \mathrm{~m}^{2}$ group was $1.376(95 \% \mathrm{Cl} 1.301-1.451)$, which was significantly higher than that (1.250) for the eGFR $60-89 \mathrm{ml} \mathrm{min}^{-1}$ per $1.73 \mathrm{~m}^{2}$ group $(95 \% \mathrm{Cl} 1.215-1.285)(P=0.009)$ and tended to be higher than that $(1.264)$ for the eGFR $\geqslant 90 \mathrm{ml} \mathrm{min}^{-1}$ per $1.73 \mathrm{~m}^{2}$ group (95\% Cl 1.188-1.340) (analysis of covariance, adjusted for age). The present study showed that CKD and subclinical lacunar infarction independently contributed to frontal lobe dysfunction in healthy elderly subjects.
\end{abstract}

Hypertension Research (2011) 34, 1023-1028; doi:10.1038/hr.2011.83; published online 23 June 2011

Keywords: glomerular filtration rate; magnetic resonance imaging; silent stroke; small vessel disease; vascular cognitive impairment

\section{INTRODUCTION}

Chronic kidney disease (CKD) is associated with an increased risk for development of cardiovascular diseases. ${ }^{1,2}$ Graded association was observed between a reduced estimated glomerular filtration rate (eGFR) and the risk of all-cause death and cardiovascular events independent of known risk factors in large community-based populations. ${ }^{3,4}$ The Chronic Kidney Disease Prognosis Consortium reported the results of a collaborative meta-analysis, including 1.23 million participants, with a median follow-up of 7.9 years and showed that eGFR and albuminuria were associated with all-cause mortality and cardiovascular mortality independent of each other and traditional cardiovascular risk factors. ${ }^{5}$ Microalbuminuria and low GFR were strongly associated with incident stroke risk. ${ }^{6,7}$ Cystatin $\mathrm{C}$ has been proposed as a better marker of kidney function than serum creatinine. In the Modification of Diet in Renal Disease Study, which included a cohort of patients with stage 3 or $4 \mathrm{CKD}$, serum cystatin C levels robustly correlated with all-cause and cardiovascular mortality. ${ }^{8}$ The mechanisms that lead to increased cardiovascular event risk include hypertension, dyslipidemia, activation of the renin-angiotensin system, endothelial dysfunction, and the effects of asymmetric dimethyl arginine, oxidative stress and inflammation. ${ }^{9}$

$\mathrm{CKD}$ is an independent risk factor for cognitive impairment, dementia and vascular cognitive impairment in population-based cohorts. ${ }^{10-14}$ CKD has been associated with lower test scores of global cognitive function, executive function, language and memory. ${ }^{15-17}$ The cause of cognitive impairment in subjects with CKD is not

\footnotetext{
${ }^{1}$ Center for Emotional and Behavioral Disorders, National Hospital Organization Hizen Psychiatric Center, Saga, Japan; ${ }^{2}$ Department of Clinical Pharmacology, Graduate School of Medical Sciences, Kyushu University, Fukuoka, Japan; ${ }^{3}$ Division of Biostatistics, Kurume University School of Medicine, Fukuoka, Japan; ${ }^{4}$ Life Science Systems Department, Fujitsu Kyushu Systems Ltd., Fukuoka, Japan and ${ }^{5}$ Department of Radiology, Saitama Medical University International Medical Center, Saitama, Japan ${ }^{6}$ Koji Yahara carried out the biostatistical analysis.

Correspondence: Dr H Yao, Center for Emotional and Behavioral Disorders, National Hospital Organization Hizen Psychiatric Center, Mitsu 160, Yoshinogari, Kanzaki, Saga 842-0192, Japan. 
clear. MRI studies have found that subjects with CKD, defined by reduced creatinine clearance, reduced eGFR or albuminuria, had a greater burden of cerebral small vessel disease (that is, lacunar infarction and leukoaraiosis) after adjusting for confounders such as vascular risk factors. ${ }^{18-22}$ It is possible that vascular factors and/or cerebral ischemic lesions may be the basis for cognitive impairment (that is, vascular cognitive impairment) in subjects with CKD. Vascular dementia, or vascular cognitive impairment, disproportionately affects frontal systems: subcortical ischemic lesions, particularly lacunar infarcts, correlated with executive dysfunction or hypometabolism in the dorsolateral frontal cortex. ${ }^{23-25}$ However, it is not clear whether CKD causes cognitive dysfunction independently of subclinical ischemic lesions on MRI. Therefore, we conducted a populationbased, cross-sectional analysis of brain-MRI findings to examine the relationship between the following: (1) subclinical MRI lesions, (2) vascular risk factors, including $\mathrm{CKD}$, and (3) cognitive function, particularly frontal lobe dysfunction.

\section{METHODS}

\section{Participants}

Between 1997 and 2009, we randomly contacted approximately 1200 inhabitants, aged $\geqslant 40$ years, living in the rural community of Sefuri village, Saga, Japan, through the village office information. Ultimately, we enrolled 754 subjects (63\%) for their first MRI examination. These subjects were living independently at home without apparent dementia. In the present study, we examined 548 elderly subjects aged $\geqslant 60$ years. Three subjects did not undergo MRI examination, because of claustrophobia or contraindications for MRI. Two subjects who did not have a serum creatinine measurement were excluded. Subjects with no modified Stroop test (MST) $(n=17)$, lack of documented educational level $(n=3)$, history of a self-reported or verified stroke $(n=14)$, psychiatric disorders including depression $(n=4)$ or a history of head trauma $(n=2)$ were also excluded. Subjects with a history of minor stroke and normal neurological examination upon MRI imaging were included. The National Hospital Organization Hizen Psychiatric Center Institutional Review Board approved the study (No. 15-1), and written informed consent was obtained from all subjects.

\section{Assessment of cognitive function}

All participants underwent a structured clinical interview. For the neurological examination, the presence or absence of a Barre sign, asymmetric deep tendon reflexes, and Babinski's sign were recorded on each side. All subjects underwent the Mini Mental State Examination (MMSE) as a global measure and MST for executive or frontal lobe function as previously described (Supplementary
Figure 1). ${ }^{26,27}$ The difference in time between the two parts was considered, owing to color interference. The subject was judged as having frontal lobe dysfunction if the total score was above the fifth quintile for each given decade as follows: $\geqslant 27 \mathrm{~s}$ for $60-69$ years, $\geqslant 30 \mathrm{~s}$ for $70-79$ years and $\geqslant 56 \mathrm{~s}$ for $80-93$ years. The subject was judged as having global cognitive dysfunction if the total MMSE score was in the lowest quintile for each given decade as follows: $<26$ for 60-69 years, $<25$ for $70-79$ years and $<23$ for $80-93$ years. As the distribution of Stroop test scores was skewed, the scores were logarithmically transformed for analysis of covariance (Figure 1).

\section{Definition of vascular risk factors}

Fasting blood samples were taken in the morning, and general hematology and biochemistry tests were performed. Blood pressure was measured in the sitting position by the standard cuff method after a 5-min rest. Vascular risk factors were defined as previously described. ${ }^{28}$ Briefly, arterial hypertension was considered as present if a subject had a history of repeated blood pressure recordings above $140 / 90 \mathrm{~mm} \mathrm{Hg}$ or the subject was being treated for hypertension. Diabetes mellitus was defined as having a fasting plasma glucose level $>7.77 \mathrm{mmoll}^{-1}$ and/or HbAlc $>6.0 \%$ or a previous diagnosis of diabetes mellitus. Hyperlipidemia was defined by either total serum cholesterol concentration greater than $5.69 \mathrm{mmoll}^{-1}$ or current treatment for hyperlipidemia. Left ventricular hypertrophy, ST depression and atrial fibrillation were measured on 12 lead electrocardiograms. We obtained information about usual alcohol intake and type of alcohol consumed from a detailed questionnaire. In the present study, we defined alcohol intake as one drink or more per week, and former drinkers were considered as non-drinkers. Smoking was defined as present if the subject smoked at least an average of 10 cigarettes per day.

Serum creatinine values, measured by the enzymatic method, were used for the new Japanese equation of eGFR modified from the Modification of Diet in Renal Disease Study equation: eGFR $\left(\mathrm{mL} \mathrm{min}^{-1}\right.$ per $\left.1.73 \mathrm{~m}^{2}\right)=194 \times($ serum creatinine $\left.\left[\mathrm{mg} \mathrm{dl}^{-1}\right]\right)^{-1.094} \times(\text { age })^{-0.287} \times(0.739$ if female $){ }^{29}$ GFR levels were classified as $\geqslant 90 \mathrm{ml} \mathrm{min}^{-1}$ per $1.73 \mathrm{~m}^{2}, 60-89 \mathrm{ml} \mathrm{min}^{-1}$ per $1.73 \mathrm{~m}^{2}$ and $<60 \mathrm{ml} \mathrm{min}^{-1}$ per $1.73 \mathrm{~m}^{2}$ according to the standard guidelines. ${ }^{1,2}$ There were no subjects with stage $5 \mathrm{CKD}$.

\section{Assessment of MRI findings}

The combination of T1-weighted (T1WI), T2-weighted (T2WI) and fluid attenuated inversion recovery images is required to accurately detect both silent brain infarction (SBI) and white matter lesions (WMLs). ${ }^{30}$ Therefore, T1WI $(\mathrm{TR} / \mathrm{TE}=510 / 12 \mathrm{~ms}), \mathrm{T} 2 \mathrm{WI} \quad(\mathrm{TR} / \mathrm{TE}=4300 / 110 \mathrm{~ms})$, and fluid attenuated inversion recovery $(\mathrm{TR} / \mathrm{TI} / \mathrm{TE}=6750 / 1600 / 22 \mathrm{~ms})$ images were obtained with a slice thickness of $6 \mathrm{~mm}$ with a $1-\mathrm{mm}$ interslice gap (device: MRI $1.0 \mathrm{~T}$, Shimadzu Magnex XP, Kyoto, Japan). SBI appeared as low signal intensities on T1-weighted images, and their size was $\geqslant 5 \mathrm{~mm}$ as previously described. ${ }^{28}$ We differentiated enlarged perivascular spaces from SBI based on their location, a

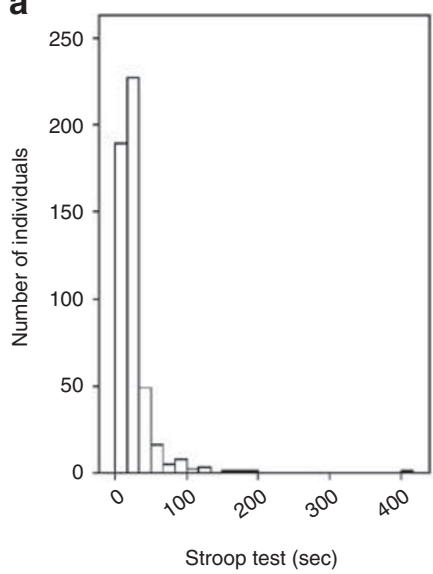

b

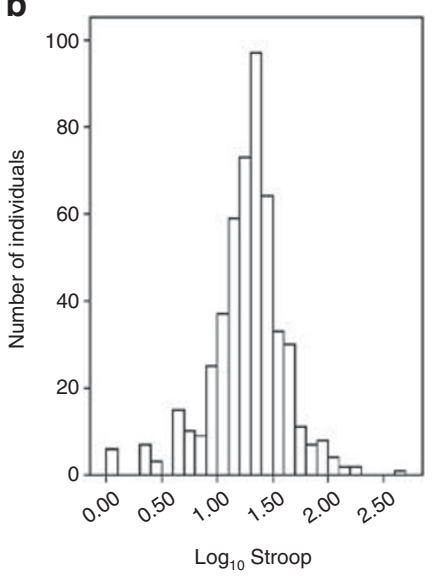

C

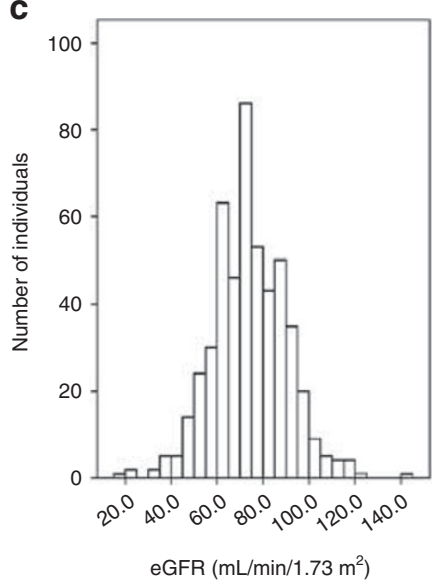

Figure 1 Distribution of modified Stroop test (MST) scores (a), logarithmically transformed score of MST (b) and estimated glomerular filtration rate (eGFR) (c). The logarithmically transformed MST scores and eGFR were distributed near normally. 
shape and size. The WMLs were defined as isointense to normal brain parenchyma on T1-weighted images, but were high-signal-intensity areas on T2-weighted images. These were classified into either deep white matter lesions (DWMLs) or periventricular hyperintensities. We used the validated rating scale of DWMLs by Fazekas et al.: grade 0, absent; grade 1, punctate foci; grade 2 , beginning confluence of foci; and grade 3 , large confluent areas. ${ }^{31}$ For periventricular hyperintensities, we determined the presence and severity (grade 0 , absent; grade 1, pencil thin; grade 2, smooth halo lining) using fluid attenuated inversion recovery images. For statistical analyses, we used combined DWML and periventricular hyperintensity grades to evaluate WMLs. All scans were reviewed independently by two authors (HY and AU) who were blinded to all clinical data. In the case of disagreement between the raters, a consensus reading was held.

\section{Statistical analysis}

Summary statistics of clinical variables were given as the median with interquartile range or the mean \pm s.d. For the univariate analysis, the nonparametric Mann-Whitney $U$-test for continuous variables was used, and the $\chi^{2}$-test for categorical variables was used to investigate differences between groups. We chose the variables for entry into the multivariate analysis based on the clinical and neuroradiological findings after univariate testing. Multivariate analysis was done using the forward stepwise method of logistic analysis. The logarithmic-transformed Stroop test scores were compared against the eGFR categories with analysis of covariance. A significance level of 0.05 was used in all analyses. The data were analyzed with the IBM SPSS Statistics 18 software for Windows (SPSS Japan).

\section{RESULTS}

The subjects were 192 men and 311 women with a mean age of 72.4 (range 60-93) years. Hypertension was present in 257 subjects $(51.1 \%)$. Blood pressure levels were $130.2 \pm 15.9 / 76.1 \pm 8.7$, $149.6 \pm 21.1 / 80.2 \pm 10.7$ and $168.5 \pm 16.1 / 87.0 \pm 9.9 \mathrm{~mm} \mathrm{Hg}$ in normotensive subjects $(n=246)$, treated hypertensive subjects $(n=198)$ and non-treated hypertensive subjects $(n=59)$, respectively. The prevalence of diabetes mellitus, hyperlipidemia and ischemic heart disease was $10.9 \%, 22.7 \%$ and $10.7 \%$, respectively. The mean value of serum creatinine was $62.8 \mu \mathrm{moll}^{-1}$ (range $35.4-255.5 \mu \mathrm{moll}^{-1}$ ), and the frequency of eGFR $<60 \mathrm{ml} \mathrm{min}^{-1}$ per $1.73 \mathrm{~m}^{2}$ was $16.5 \%$ (Figure 1c). Subclinical lacunar infarction, DWMLs and periventricular hyperintensities were detected in 79 (15.7\%), $216(42.9 \%)$ and $150(29.8 \%)$ of the 503 participants, respectively. Of the 79 subjects with subclinical lacunar infarction, 46 subjects (58.2\%) had a single lacunar infarct. There were a total of 137 lacunae in 79 subjects: 73 were located in the basal ganglia, 15 in the thalamus, 39 in the corona radiata, 4 in the cerebellum and 6 in the brainstem. The number of infarcts in the cerebellum and brainstem was operationally counted as 0 for the logistic analysis, because infratentorial lacunae are considered not to influence cortical function. Of the 216 subjects with DWMLs, small punctate lesions $(n=152)$ were the most prevalent, followed by early confluent $(n=53)$ and large confluent $(n=11)$ lesions.

The frontal lobe dysfunction group, defined as the highest quintile of Stroop test scores, tended to have higher BP (146/80 vs.

Table 1 Clinical and neuroradiological features of subjects with or without frontal lobe dysfunction

\begin{tabular}{|c|c|c|c|}
\hline & \multicolumn{2}{|c|}{ Frontal lobe dysfunction } & \multirow[b]{2}{*}{ P-value } \\
\hline & $(+) n=100$ & $(-) n=403$ & \\
\hline Age, median (IQR), years & $73(68-78)$ & $72(66-78)$ & NS \\
\hline Male sex, $n(\%)$ & $45(45.0)$ & $147(36.5)$ & 0.116 \\
\hline Education, median (IQR), years & $8(8-10)$ & $9(8-11)$ & 0.043 \\
\hline Mini Mental State Examination, median (IQR) & $26(24-28)$ & $28(26-29)$ & 0.000 \\
\hline Body mass index, median (IQR), $\mathrm{kg} \mathrm{m}^{-2}$ & $22.7(19.9-25.3)$ & $22.6(20.4-25.0)$ & NS \\
\hline Hypertension, n (\%) & $55(55.0)$ & $202(50.1)$ & NS \\
\hline Systolic BP, median (IQR), mm Hg & $146(131-162)$ & $140(126-156)$ & 0.051 \\
\hline Diastolic BP, median (IQR), $\mathrm{mm} \mathrm{Hg}$ & $80(73-88)$ & $78(72-86)$ & 0.130 \\
\hline Diabetes mellitus, $n(\%)$ & $11(11.0)$ & $44(10.9)$ & NS \\
\hline Hyperlipidemia, $n(\%)$ & $18(18.0)$ & $96(23.8)$ & NS \\
\hline Alcohol, $n(\%)$ & $30(30.0)$ & $103(25.6)$ & NS \\
\hline Smoking, $n(\%)$ & $15(15.0)$ & $42(10.4)$ & NS \\
\hline Ischemic heart disease, $n(\%)$ & $10(10.0)$ & $44(10.9)$ & NS \\
\hline Atrial fibrillation, $n(\%)$ & $1(1.0)$ & $11(2.7)$ & NS \\
\hline Hematocrit, median (IQR), fraction of 1.00 & $0.39(0.36-0.42)$ & $0.40(0.37-0.43)$ & 0.159 \\
\hline Albumin, median (IQR), $\mathrm{gl}^{-1}$ & $43(41-45)$ & $43(41-45)$ & NS \\
\hline Fasting blood glucose, median (IQR), mmol ${ }^{-1}$ & $5.16(4.83-5.59)$ & $5.22(4.88-5.66)$ & NS \\
\hline Total cholesterol, median (IQR), mmol I-1 & $5.06(4.37-5.63)$ & $5.07(4.45-5.66)$ & NS \\
\hline HDL cholesterol, median (IQR), mmol I-1 & $1.55(1.29-1.76)$ & $1.50(1.22-1.76)$ & NS \\
\hline Uric acid, median (IQR), $\mu \mathrm{mol} \mathrm{I}^{-1}$ & $300(256-363)$ & $291(238-351)$ & 0.188 \\
\hline Creatinine, median (IQR), $\mu \mathrm{mol} \mathrm{I}^{-1}$ & $62.8(53.0-71.6)$ & $60.1(52.2-69.8)$ & 0.000 \\
\hline eGFR, median (IQR), $\mathrm{ml} \mathrm{min}^{-1}$ per $1.73 \mathrm{~m}^{2}$ & $71.4(60.9-82.3)$ & $74.4(64.2-85.8)$ & 0.012 \\
\hline \multicolumn{4}{|l|}{ MRI findings } \\
\hline Subclinical lacunar infarction, $n(\%)$ & $24(24.0)$ & $55(13.6)$ & 0.011 \\
\hline Deep white-matter lesions, $n(\%)$ & $50(50.0)$ & $166(41.2)$ & 0.111 \\
\hline Periventricular hyperintensities, $n(\%)$ & $37(37.0)$ & $113(28.0)$ & 0.080 \\
\hline
\end{tabular}


Table 2 Logistic regression analysis of factors relevant to frontal lobe dysfunction

\begin{tabular}{|c|c|c|c|c|c|c|c|}
\hline & \multirow{2}{*}{$\begin{array}{c}\text { Univariate } \\
\mathrm{P}\end{array}$} & \multicolumn{3}{|c|}{ Multivariate: Model 1} & \multicolumn{3}{|c|}{ Multivariate: Model 2} \\
\hline & & $O R$ & $95 \% \mathrm{Cl}$ & $\mathrm{P}$ & $O R$ & $95 \% \mathrm{Cl}$ & $P$ \\
\hline Male sex & 0.116 & 1.615 & $1.023-2.548$ & 0.039 & & & \\
\hline Education (per year) & 0.087 & & & & & & \\
\hline Diastolic BP $\left(\mathrm{mm}^{-1} \mathrm{Hg}\right)$ & 0.098 & & & & & & \\
\hline White matter lesions & 0.061 & & & & & & \\
\hline
\end{tabular}

Abbreviations: BP, blood pressure; eGFR, estimated glomerular filtration rate; No., number; OR, odds ratio.

Model 1: age, sex, education, systolic and diastolic BP, and eGFR were included in the forward stepwise method of logistic analysis. Model 2: MRI findings were added to Model 1.

140/78 $\mathrm{mm} \mathrm{Hg}$ ), less education (median 8 vs. 9 years), higher creatinine $\left(62.8 v\right.$ vs. $\left.60.1 \mu \mathrm{moll}^{-1}\right)$ and lower eGFR $\left(71.4\right.$ vs. $74.4 \mathrm{ml} \mathrm{min}^{-1}$ per $1.73 \mathrm{~m}^{2}$ ) (Table 1). When possible confounders were entered into the multivariate logistic regression model (the forward stepwise method), the independent predictors of frontal lobe dysfunction were male sex (odds ratio 1.615; 95\% CI 1.023-2.548) and eGFR (odds ratio 0.816 ; $95 \%$ CI $0.708-0.940$ per $10 \mathrm{ml} \mathrm{min}^{-1}$ per $1.73 \mathrm{~m}^{2}$ ) in model 1 (Table 2). Although frontal lobe dysfunction was more prevalent among men in model $1(P=0.039)$, this sex difference disappeared in model 2 after adjustment for MRI findings: the independent predictors of frontal lobe dysfunction were eGFR (odds ratio 0.854 ; $95 \%$ CI $0.743-0.983$ per $10 \mathrm{ml} \mathrm{min}^{-1}$ per $1.73 \mathrm{~m}^{2}$ ) and the number of lacunar infarction (odds ratio 1.460; 95\% CI 1.127-1.892). When eGFR was replaced with the presence of CKD, this sex difference disappeared even in model 1; the other results were essentially the same as those in model 2. If diastolic blood pressure was included in model 2, diastolic blood pressure tended to be associated with frontal lobe dysfunction, but did not reach statistical significance $(P=0.054)$. When MMSE score was replaced with MST as a dependent variable in model 2, neither the CKD nor the number of lacunar infarction was associated with cognitive dysfunction, defined as a patient testing in the lowest quintile of MMSE scores for each given decade (data not shown). In contrast, cognitive dysfunction defined by MMSE was significantly associated with education (odds ratio 0.665 ; 95\% CI $0.569-0.776$ per year) and the combined grade of WMLs (odds ratio 1.324; 95\% CI 1.085-1.616).

The Stroop test score was compared against the eGFR categories using analysis of covariance adjusted for age (Figure 2). The assumption regression slope homogeneity was tested $(P=0.124)$. The mean of the logarithmic-transformed MST scores in the eGFR $<60 \mathrm{ml} \mathrm{min}^{-1}$ per $1.73 \mathrm{~m}^{2}$ group was 1.376 (95\% CI 1.301-1.451), which was significantly higher than 1.250 (95\% CI 1.215-1.285) for the eGFR $60-89 \mathrm{ml} \mathrm{min}^{-1}$ per $1.73 \mathrm{~m}^{2}$ group $(P=0.009$, adjusted for multiple comparisons with Bonferroni correction) and tended to be higher than $1.264(95 \%$ CI $1.188-1.340)$ in the $\geqslant 90 \mathrm{ml} \mathrm{min}^{-1}$ per $1.73 \mathrm{~m}^{2}$ group. The MST scores among the eGFR categories were essentially the same after further adjustment for education (data not shown).

\section{DISCUSSION}

The present study showed that subclinical lacunar infarction and CKD were independently associated with frontal lobe dysfunction in healthy elderly people, while WMLs were related to global cognitive dysfunction. We have reported a male predominance in SBI, which was largely due to a higher prevalence of alcohol intake and smoking in men than women in our population. ${ }^{28}$ In the same context, frontal lobe

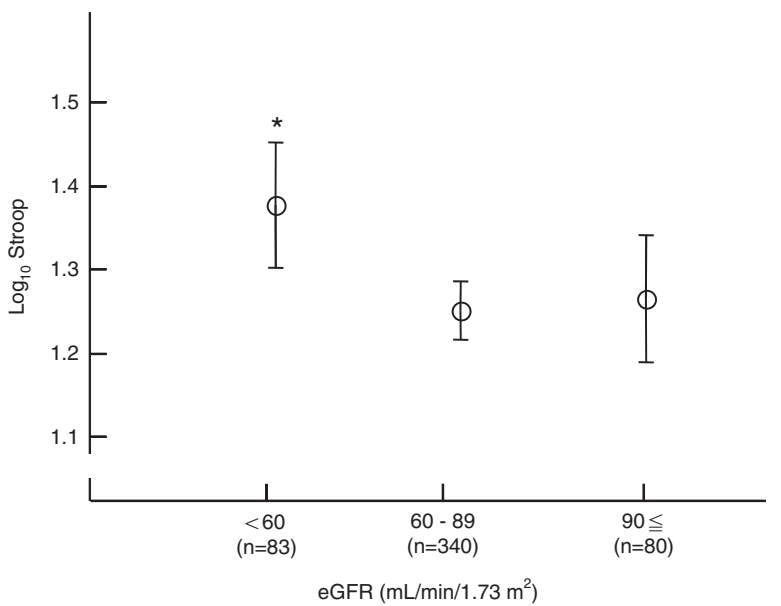

Figure 2 Logarithmically transformed score of modified Stroop test (MST) according to the estimated glomerular filtration rate (eGFR) category. Graph shows mean values and $95 \%$ confidence interval of logarithmically transformed score of MST adjusted for age. ${ }^{*} P=0.009$ vs. eGFR $60-89 \mathrm{ml} \mathrm{min}^{-1}$ per $1.73 \mathrm{~m}^{2}$.

dysfunction was related to male sex in model 1 but not in the final multivariate model that included MRI findings (model 2). Subcortical vascular disease and DWMLs seen in elderly subjects were associated with cognitive impairment or dementia, depression, apathy and gait disturbance. $^{23,32,33}$ Multiple lacunae have been known to cause frontal lobe dysfunction, including difficulty in set shifting, impaired executive function and decreased verbal fluency, as seen in the present study. ${ }^{24,25,27}$ This is the first study that showed CKD was associated with frontal lobe dysfunction independently of subclinical lacunar stroke.

One major limitation of this study relates to its cross-sectional study design, which limits the interpretation of our results with respect to cause and effect, particularly CKD as a presumed prerequisite for subsequent cognitive decline. However, we cannot exclude the possibility that CKD-associated factors concurrently affect frontal lobe function. Another limitation is the narrow scope of the cognitive assessment, because only MST and MMSE were performed. The use of broader testing would have produced more in-depth evaluation of neuropsychological characteristics in relation to CKD. The definition of 'frontal lobe dysfunction' by only one assessment battery is also a limitation of our study. Finally, neither urinary albumin nor cystatin C was measured in the present study, which may have limited the evaluation of kidney function. It is, however, noteworthy that eGFR 
are the best overall indices of kidney function. ${ }^{1}$ The advantages of the current study are the inclusion of a relatively large number of community-dwelling subjects and the use of MRI. In particular, structural brain imaging with MRI is indispensable for assessing the underlying mechanism of cognitive dysfunction associated with CKD.

There is mounting evidence that CKD increases the risk of cognitive decline and dementia. During a median 6-year follow-up of community-dwelling older adults, moderate renal insufficiency was independently associated with a $37 \%$ increased risk of incident dementia, particularly vascular-type dementia. ${ }^{10}$ Cognitive impairment in subjects with CKD is mediated by the direct effects of uremia or is attributable to a high prevalence of vascular risk factors. ${ }^{11}$ Most likely, CKD may exert adverse effects on cerebral vasculature and cause vascular cognitive impairment. ${ }^{12}$ In a large cohort of elderly subjects, moderate-to-severe impairment of renal function, defined as creatinine clearance $<45 \mathrm{ml} \mathrm{min}^{-1}$ per $1.73 \mathrm{~m}^{2}$, was associated with newly diagnosed cognitive impairment after 2 years. ${ }^{13}$ Similarly, older community-dwelling men with albuminuria had worse cognitive function and greater cognitive decline over a 6.6-year interval. ${ }^{14}$ Cognitive function measured across multiple domains, including executive function, was worse in subjects with more severe kidney function. ${ }^{15,17}$ Specific cognitive domains were more vulnerable to moderate CKD. These included visual-spatial organization and memory, scanning and tracking abilities, which are mostly executive skills. ${ }^{16}$ Frontal lobe dysfunction is a feature of vascular dementia or vascular cognitive impairment. ${ }^{23}$ Our present study provided further evidence that CKD may be an independent risk factor for frontal rather than global cognitive dysfunction, suggesting that $\mathrm{CKD}$ acts as a vascular factor. This study cannot explain the mechanism of observed association of CKD and frontal lobe dysfunction, which was independent of lacunar infarction. Knopman ${ }^{34}$ proposed that microvascular disease or endothelial dysfunction addresses at least one way that CKD causes cognitive decline. In contrast, Gouw et al. ${ }^{35}$ emphasized that lesions invisible on MRI, such as cortical microinfarcts and tissue changes in the normal-appearing white matter, may have an important clinical role as major determinants of cognitive decline.

Additionally, CKD-associated ischemic brain lesions may be the basis for cognitive decline. The Northern Manhattan Study and the Rotterdam Scan Study found that subjects with CKD, defined by reduced creatinine clearance or eGFR, had a greater burden of white matter hyperintensity volumes after adjusting for confounders such as vascular risk factors. ${ }^{19,20}$ Likewise, in community-dwelling Japanese elderly subjects, the association between the presence of CKD and lacunar infarction or moderate WMLs was statistically significant even after adjustment for conventional cardiovascular risk factors, suggesting that $\mathrm{CKD}$ was an independent risk factor for cerebral small vessel disease. ${ }^{21}$ Albuminuria was associated with worse executive function, as well as increased white-matter-hyperintensity volumes in homebound elders. ${ }^{22}$ Further, cystatin C, a cysteine proteinase inhibitor, has been proposed as an alternative and more accurate renal function marker. Increasing prevalence of SBI was associated independently with increasing quintiles of cystatin $\mathrm{C}$ range, whereas the associations between serum creatinine or eGFR and SBI were U-shaped. ${ }^{18}$ In the present study, however, CKD was not significantly associated with subclinical lacunar infarction in the multivariate logistic regression model. One possible explanation for this negative relationship could be the cross-sectional design of our study. CKD and lacunar infarction were simultaneously and independently associated with frontal but not global cognitive dysfunction.

There are several biologically plausible mechanisms through which CKD could result in subclinical MRI lesions and cognitive decline.
Although folic acid supplementation significantly improved cognitive function, ${ }^{36}$ the renal Heart Outcomes Prevention Evaluation-2 study recently reported that active treatment with $\mathrm{B}$ vitamins lowered homocysteine in participants with CKD but had no effect on cardiovascular outcomes. ${ }^{37}$ High rather than low adiponectin, an adipocyte-derived protein with anti-inflammatory, anti-atherogenic and insulin-sensitizing activity, was a predictor of all-cause and cerebrovascular mortality in patients with stage $3-4$ CKD. ${ }^{38}$ There have been no interventional studies with adiponectin on cardiovascular outcome; thus, it remains unclear whether adiponectin confers vascular protection. ${ }^{39}$ Inflammatory and procoagulant pathways and asymmetric dimethylarginine, an endogenous inhibitor of nitric oxide synthase, might be important mediators leading to the increased cardiovascular risks of patients with CKD. ${ }^{40,41}$ Anemia is another contributing factor of cognitive dysfunction in hemodialysis patients, based on the observation that graded increments in hematocrit following erythropoietin therapy improved neuropsychological tests as well as eventrelated potential. ${ }^{42}$ However, in the present study with healthy elderly subjects, hematocrit was not associated with cognitive function.

In conclusion, the present study showed that CKD and subclinical lacunar infarction were independently associated with frontal lobe dysfunction in healthy elderly subjects. In other words, subjects with $\mathrm{CKD}$ have an increased risk of developing vascular cognitive impairment. Interventions directed at treatment and prevention of CKD, as well as silent ischemic brain lesions, particularly lacunar infarcts, would be beneficial in attenuating frontal cognitive decline in healthy elderly subjects.

\section{CONFLICT OF INTEREST}

The authors declare no conflict of interest.

\section{ACKNOWLEDGEMENTS}

We wish to express special thanks to T Ninomiya, MD, for his valuable advice during the course of this study. We also thank T Muto, K Imada and $\mathrm{K}$ Yamamoto for technical assistance with the laboratory examinations and MRI scanning, and N Kawahara-Ideno and $\mathrm{K}$ Muto for registration of participants.

1 Levey AS, Coresh J, Balk E, Kausz AT, Levin A, Steffes MW, Hogg RJ, Perrone RD, Lau J, Eknoyan G, National Kidney Foundation. National Kidney Foundation practice guidelines for chronic kidney disease: evaluation, classification, and stratification. Ann Intern Med 2003; 139: 137-147.

2 Sarnak MJ, Levey AS, Schoolwerth AC, Coresh J, Culleton B, Hamm LL, McCullough PA, Kasiske BL, Kelepouris E, Klag MJ, Parfrey P, Pfeffer M, Raij L, Spinosa DJ, Wilson PW, American Heart Association Councils on Kidney in Cardiovascular Disease, High Blood Pressure Research, Clinical Cardiology, and Epidemiology and Prevention. Kidney disease as a risk factor for development of cardiovascular disease: a statement from the American Heart Association Councils on Kidney in Cardiovascular Disease, High Blood Pressure Research, Clinical Cardiology, and Epidemiology and Prevention. Circulation 2003; 108: 2154-2169.

3 Go AS, Chertow GM, Fan D, McCulloch CE, Hsu CY. Chronic kidney disease and the risks of death, cardiovascular events, and hospitalization. N Engl J Med 2004; 351: 1296-1305.

4 Ninomiya T, Kiyohara Y, Tokuda Y, Doi Y, Arima H, Harada A, Ohashi Y, Ueshima H, Japan Arteriosclerosis Longitudinal Study Group. Impact of kidney disease and blood pressure on the development of cardiovascular disease: an overview from the Japan Arteriosclerosis Longitudinal Study. Circulation 2008; 118: 2694-2701.

5 Chronic Kidney Disease Prognosis ConsortiumMatsushita K, van der Velde M, Astor BC, Woodward M, Levey AS, de Jong PE, Coresh J, Gansevoort RT. Association of estimated glomerular filtration rate and albuminuria with all-cause and cardiovascular mortality in general population cohorts: a collaborative meta-analysis. Lancet 2010; 375: 20732081.

6 Lee M, Saver JL, Chang KH, Liao HW, Chang SC, Ovbiagele B. Impact of microalbuminuria on incident stroke: a meta-analysis. Stroke 2010; 41: 2625-2631.

7 Lee M, Saver JL, Chang KH, Liao HW, Chang SC, Ovbiagele B. Low glomerular filtration rate and risk of stroke: meta-analysis. Br Med J 2010; 341: c4249. 
8 Menon V, Shlipak MG, Wang X, Coresh J, Greene T, Stevens L, Kusek JW, Beck GJ, Collins AJ, Levey AS, Sarnak MJ. Cystatin C as a risk factor for outcomes in chronic kidney disease. Ann Intern Med 2007; 147: 19-27.

9 Schiffrin EL, Lipman ML, Mann JF. Chronic kidney disease: effects on the cardiovascular system. Circulation 2007; 116: 85-97.

10 Seliger SL, Siscovick DS, Stehman-Breen CO, Gillen DL, Fitzpatrick A, Bleyer A, Kuller LH. Moderate renal impairment and risk of dementia among older adults: the Cardiovascular Health Cognition Study. J Am Soc Nephrol 2004; 15: 1904-1911.

11 Kurella M, Chertow GM, Fried LF, Cummings SR, Harris T, Simonsick E, Satterfield S, Ayonayon $\mathrm{H}$, Yaffe $\mathrm{K}$. Chronic kidney disease and cognitive impairment in the elderly: the health, aging, and body composition study. J Am Soc Nephrol 2005; 16 : 2127-2133.

12 Khatri M, Nickolas T, Moon YP, Paik MC, Rundek T, Elkind MS, Sacco RL, Wright CB. CKD associates with cognitive decline. J Am Soc Nephrol 2009; 20: 2427-2432.

13 Etgen T, Sander D, Chonchol M, Briesenick C, Poppert H, Förstl H, Bickel H. Chronic kidney disease is associated with incident cognitive impairment in the elderly: the INVADE study. Nephrol Dial Transplant 2009; 24: 3144-3150.

14 Jassal SK, Kritz-Silverstein D, Barrett-Connor E. A prospective study of albuminuria and cognitive function in older adults: the Rancho Bernardo study. Am J Epidemiol 2010; 171: 277-286.

15 Buchman AS, Tanne D, Boyle PA, Shah RC, Leurgans SE, Bennett DA. Kidney function is associated with the rate of cognitive decline in the elderly. Neurology 2009; 73: 920-927.

16 Elias MF, Elias PK, Seliger SL, Narsipur SS, Dore GA, Robbins MA. Chronic kidney disease, creatinine and cognitive functioning. Nephrol Dial Transplant 2009; 24: 2446-2452.

17 Yaffe K, Ackerson L, Kurella Tamura M, Le Blanc P, Kusek JW, Sehgal AR, Cohen D, Anderson C, Appel L, Desalvo K, Ojo A, Seliger S, Robinson N, Makos G, Go AS, Chronic Renal Insufficiency Cohort Investigators. Chronic kidney disease and cognitive function in older adults: findings from the chronic renal insufficiency cohort cognitive study. J Am Geriatr Soc 2010; 58: 338-345.

18 Seliger SL, Longstreth Jr WT, Katz R, Manolio T, Fried LF, Shlipak M, Stehman-Breen CO, Newman A, Sarnak M, Gillen DL, Bleyer A, Siscovick DS. Cystatin C and subclinical brain infarction. J Am Soc Nephrol 2005; 16: 3721-3727.

19 Khatri M, Wright CB, Nickolas TL, Yoshita M, Paik MC, Kranwinkel G, Sacco RL, DeCarli C. Chronic kidney disease is associated with white matter hyperintensity volume: the Northern Manhattan Study (NOMAS). Stroke 2007; 38: 3121-3126.

20 Ikram MA, Vernooij MW, Hofman A, Niessen WJ, van der Lugt A, Breteler MM. Kidney function is related to cerebral small vessel disease. Stroke 2008; 39: 55-61.

21 Wada M, Nagasawa H, Iseki C, Takahashi Y, Sato H, Arawaka S, Kawanami T, Kurita K, Daimon M, Kato T. Cerebral small vessel disease and chronic kidney disease (CKD): results of a cross-sectional study in community-based Japanese elderly. J Neurol Sci 2008; 272: 36-42.

22 Weiner DE, Bartolomei K, Scott T, Price LL, Griffith JL, Rosenberg I, Levey AS, Folstein MF, Sarnak MJ. Albuminuria, cognitive functioning, and white matter hyperintensities in homebound elders. Am J Kidney Dis 2009; 53: 438-447.

23 O'Brien JT, Erkinjuntti T, Reisberg B, Roman G, Sawada T, Pantoni L, Bowler JV, Ballard C, DeCarli C, Gorelick PB, Rockwood K, Burns A, Gauthier S, DeKosky ST. Vascular cognitive impairment. Lancet Neurol 2003; 2: 89-98.

24 Reed BR, Eberling JL, Mungas D, Weiner M, Kramer JH, Jagust WJ. Effects of white matter lesions and lacunes on cortical function. Arch Neurol 2004; 61: 1545-1550.
25 Carey CL, Kramer JH, Josephson SA, Mungas D, Reed BR, Schuff N, Weiner MW, Chui $\mathrm{HC}$. Subcortical lacunes are associated with executive dysfunction in cognitively normal elderly. Stroke 2008; 39: 397-402.

26 MacLeod CM. Half a century of research on the Stroop effect: an integrative review. Psychol Bull 1991; 109: 163-203.

27 Takashima Y, Yao H, Koga H, Endo K, Matsumoto T, Uchino A, Sadanaga-Akiyoshi F, Yuzuriha T, Kuroda Y. Frontal lobe dysfunction caused by multiple lacunar infarction in community-dwelling elderly subjects. J Neurol Sci 2003; 214: 37-41.

28 Takashima Y, Miwa Y, Mori T, Hashimoto M, Uchino A, Yuzuriha T, Sasaguri T, Yao H. Sex differences in the risk profile and male predominance in silent brain infarction in community-dwelling elderly subjects: the Sefuri brain MRI study. Hypertens Res 2010; 33: 748-752.

29 Matsuo S, Imai E, Horio M, Yasuda Y, Tomita K, Nitta K, Yamagata K, Tomino Y, Yokoyama $\mathrm{H}$, Hishida A. Revised equations for estimated GFR from serum creatinine in Japan. Am J Kidney Dis 2009; 53: 982-992.

30 Sasaki M, Hirai T, Taoka T, Higano S, Wakabayashi C, Matsusue E, Ida M. Discriminating between silent cerebral infarction and deep white matter hyperintensity using combinations of three types of magnetic resonance images: a multicenter observer performance study. Neuroradiology 2008; 50: 753-758.

31 Fazekas F, Kleinert R, Offenbacher H, Schmidt R, Kleinert G, Payer F, Radner H, Lechner H. Pathologic correlates of incidental MRI white matter signal hyperintensities. Neurology 1993; 43: 1683-1689.

32 O'Sullivan M. Leukoaraiosis. Pract Neurol 2008; 8: 26-38.

33 Yao H, Takashima Y, Mori T, Uchino A, Hashimoto M, Yuzuriha T, Miwa Y, Sasaguri T. Hypertension and white matter lesions are independently associated with apathetic behavior in healthy elderly subjects: the Sefuri brain MRI study. Hypertens Res 2009; 32: $586-590$.

34 Knopman DS. Invited commentary: Albuminuria and microvascular disease of the brain-a shared pathophysiology. Am J Epidemiol 2010; 171: 287-289.

35 Gouw AA, Seewann A, van der Flier WM, Barkhof F, Rozemuller AM, Scheltens P, Geurts JJ. Heterogeneity of small vessel disease: a systematic review of MRI and histopathology correlations. J Neurol Neurosurg Psychiatry 2011; 82: 126-135.

36 Brady CB, Gaziano JM, Cxypoliski RA, Guarino PD, Kaufman JS, Warren SR, Hartigan P, Goldfarb DS, Jamison RL. Homocysteine lowering and cognition in CKD: the Veterans Affairs homocysteine study. Am J Kidney Dis 2009; 54: 440-449.

37 Mann JF, Sheridan P, McQueen MJ, Held C, Arnold JM, Fodor G, Yusuf S, Lonn EM, HOPE-2 investigators. Homocysteine lowering with folic acid and B vitamins in people with chronic kidney disease-results of the renal Hope-2 study. Nephrol Dial Transplant 2008; 23: 645-653.

38 Menon V, Li L, Wang X, Greene T, Balakrishnan V, Madero M, Pereira AA, Beck GJ, Kusek JW, Collins AJ, Levey AS, Sarnak MJ. Adiponectin and mortality in patients with chronic kidney disease. J Am Soc Nephrol 2006; 17: 2599-2606.

39 Kaisar OM, Johnson DW, Prins JB, Isbel N. The role of novel biomarkers of cardiovascular disease in chronic kidney disease: focus on adiponectin and leptin. Curr Cardiol Rev 2008; 4: 287-292.

40 Shlipak MG, Fried LF, Crump C, Bleyer AJ, Manolio TA, Tracy RP, Furberg CD, Psaty $\mathrm{BM}$. Elevations of inflammatory and procoagulant biomarkers in elderly persons with renal insufficiency. Circulation 2003; 107: 87-92.

41 Young JM, Terrin N, Wang X, Greene T, Beck GJ, Kusek JW, Collins AJ, Sarnak MJ, Menon V. Asymmetric dimethylarginine and mortality in stages 3 to 4 chronic kidney disease. Clin J Am Soc Nephrol 2009; 4: 1115-1120.

42 Stivelman JC. Benefits of anaemia treatment on cognitive function. Nephrol Dial Transplant 2000; 15(suppl 3): 29-35.

Supplementary Information accompanies the paper on Hypertension Research website (http://www.nature.com/hr) 